\title{
How Flat is Your Detector? Non-Uniform Annular Detector Sensitivity in STEM Quantification
}

\author{
Katherine E. MacArthur, Lewys Jones and Peter D. Nellist \\ University of Oxford, Department of Materials Science, Oxford, UK
}

Quantification of high-angle annular dark-field scanning transmission electron microscopy (HAADF STEM) data has been of growing interest over the past few years. The most common approach involves normalizing the HAADF image intensity to the incident beam current to facilitate direct comparison with theoretical values [1]. This quantitative STEM imaging requires two key conditions to be met; firstly that the detector amplifier be in a linearly amplifying range, and secondly that the detector's recording sensitivity, or efficiency, must be known [2]. This sensitivity can be determined by scanning the STEM probe in a raster over the detector plane. The count-rate from regions of vacuum indicates the amplifier's D.C. offset, and the additional count-rate across the active region of the detector is then equivalent to the total current of the probe. Ideally, the efficiency of the active region would be uniform but this is rarely the case and instead an asymmetric detector map is often obtained. This can lead to errors in the normalisation factor used and subsequently to errors in the quantitative HAADF images themselves. In the quest for accurate quantitative STEM data, that can be reliably compared with simulation, it is logical then to look for the best possible behavior in our detectors.

Starting from the premise that detectors are not perfectly uniform, we first need to describe their behavior before we can make any sort of comparison. Here we evaluate the flatness (radially), roundness (azimuthally), uniformity (or smoothness) and ellipticity of six annular detectors encompassing each of the current major manufacturers, Figure 1.

For detectors whose sensitivity varies with collection angle (radially) there may be a variation in the collection-efficiency of the HAADF signal for different elements present in the sample because of the changes in the angular range of scattering. Even for pure-element specimens there may be some discrepancy between the recorded scattering and that expected from simulation. Some simulation code can now accept a vector input describing this varying 'sensitivity-profile' but it would clearly be preferable for this to be as flat as possible [3]. Detectors may also demonstrate varying sensitivity around the detector (azimuthally). In this case electron scattering over different directions may be recorded with varying efficiency - potentially leading to complicated and unwanted image artifacts [3]. These first two types of asymmetry are present in many models of detector and is often located on the side farthest from the read-out electronics. This shadow results from the physical hole (drift-tube) in the detector, which allows the bright-field and EELS signals to pass through the centre of the detector. The signal is read out to the side of the detector and regions on the far side of the drift-tube often exhibit a suppressed efficiency. Thirdly, an otherwise perfect detector may still have a random non-uniform sensitivity-texture due to the quality of finish of the active layer. Lastly, a detector can exhibit a degree of ellipticity. This can be due to detector being physically tilted within the column, a result of manufacturing error, or in systems with post-specimen aberration correction, a result of geometric distortions arising from these lower optics. Any such ellipticity will have a significant effect on the measurement of inner angle - itself a critical measurement for any comparison with simulation. For completeness the approximate outer angle (expressed as a multiple of the inner angle) is shown also. In general a large outer angle is favourable for improved total electron-scattering collection-efficiency. 


\section{References:}

[1] Singhal, A., Yang, J., \& Gibson, J. Ultramicroscopy 67 (1997), p. 1910-206.

[2] JM Lebeau, S Stemmer, Ultramicroscopy 108 (2008), p. 1653-1658.

[3] SD Findlay and JM Lebeau, Ultramicroscopy 124 (2013), p. 52-60.

[4] The authors would like to acknowledge Tim Pennycook, Armand Béché and Haibo E for assisting with the mapping, and the EPSRC and Johnson Matthey for financial support.

\begin{tabular}{|c|c|c|}
\hline Parameter & Definition & Importance \\
\hline 'Flatness' & $\begin{array}{c}\text { The consistency of sensitivity outwards with respect } \\
\text { to scattering angle (radially). Expressed here in units } \\
\text { of normalised standard-deviation }\end{array}$ & $\begin{array}{c}\text { Essential for accurate comparison with } \\
\text { simulated integrated-intensities / cross- } \\
\text { sections. }\end{array}$ \\
\hline 'Roundness' & $\begin{array}{c}\text { The consistency of sensitivity around the detector } \\
\text { (azimuthally). Expressed here in units of normalised } \\
\text { standard-deviation }\end{array}$ & $\begin{array}{c}\text { Essential for avoiding misleading } \\
\text { channeling based contrast and direct } \\
\text { interpretation of STEM images. }\end{array}$ \\
\hline 'Smoothness' & $\begin{array}{c}\text { The consistency of sensitivity of all individual points } \\
\text { on the detector. Expressed here units of in normalized } \\
\text { histogram full-width-quarter-maximum (FWQM) }\end{array}$ & $\begin{array}{c}\text { Important for the equal collection- } \\
\text { weighting of all electrons that fall on } \\
\text { the detector. }\end{array}$ \\
\hline 'Ellipticity' & $\begin{array}{c}\text { The deviation of the detector from an ideal circular } \\
\text { shape. Expressed here as the percentage of the major } \\
\text { over the minor diameters of the inner angle opening. }\end{array}$ & $\begin{array}{c}\text { Essential for having a single-reliable } \\
\text { estimate of inner angle for both } \\
\text { experiment and simulation. }\end{array}$ \\
\hline
\end{tabular}

Table 1. Summary of the symmetry parameters discussed, their definitions and their importance for reliable STEM imaging and quantification. The deviations from this symmetry are shown in Figure 1.

\begin{tabular}{|c|c|c|c|c|c|c|}
\hline \multirow{2}{*}{ Manufacturer } & \multicolumn{3}{|c|}{ Company A } & Company B & Company C & Company D \\
\hline & Microscope 1 & Microscope 2 & Microscope 3 & - & - & - \\
\hline $\begin{array}{l}\text { Detector } \\
\text { Map }\end{array}$ & & & & & & \\
\hline Outer Angle & $5.47 \mathrm{x}$ & $3.09 \mathrm{x}$ & $2.89 x$ & $5.91 x$ & $2.90 \mathrm{x}$ & $3.50 \mathrm{x}$ \\
\hline Non-flatness & $8.93 \%$ & $6.79 \%$ & $24.90 \%$ & $10.39 \%$ & $9.19 \%$ & $14.49 \%$ \\
\hline Non-roundness & $4.50 \%$ & $4.66 \%$ & $9.13 \%$ & $4.10 \%$ & $17.38 \%$ & $2.39 \%$ \\
\hline Non-smoothness & $27.5 \% *$ & $9.2 \%$ & $13.7 \%$ & $14.7 \%$ & $28.4 \%$ & $23.5 \% *$ \\
\hline Ellipticity & $19.59 \%$ & $4.77 \%$ & $8.94 \%$ & $0.49 \%$ & $4.31 \%$ & $13.30 \%$ \\
\hline Average & $15.1 \%$ & $6.4 \%$ & $14.2 \%$ & $7.4 \%$ & $13.9 \%$ & $13.4 \%$ \\
\hline
\end{tabular}

Figure 1. Scaled sensitivity images of the six detectors studied, grouped by manufacturer. For each type of non-uniformity, the percentage deviation from perfection is shown. For each performance metric the most uniform detector is highlighted in bold. Lastly, an overall "non-uniformity" score is tabulated.

* Related to hardware quality but also mapping methodology, detector saturation and sample occlusion. 